\title{
Rehabilitation medicine for elderly patients
}

\author{
Masiero $\mathrm{S}^{\mathbf{1}}$, Carraro $\mathrm{U}^{2,3,4 *}$ and Musumeci $\mathrm{A}^{\mathbf{1}}$ \\ ${ }^{1}$ Section of Physical Medicine and Rehabilitation, Department of Neuroscience, University of Padova, Italy \\ ${ }^{2}$ Interdepartmental Research Center of Myology (CIR-Myo), Department of Biomedical Science, University of Padova, Italy \\ ${ }^{3} \mathrm{~A} \& \mathrm{C}$ M-C Foundation for Translational Myology, Padova, Italy \\ ${ }^{4}$ IRCCS Fondazione Ospedale San Camillo, Venezia-Lido, Italy
}

\begin{abstract}
World older population is rapidly growing in absolute and relative number. Increased life expectancy of a large percentage of a population has many implications, specifically for older olds and their families, for the public health care system with increasing costs for managements, and for social burden. In this regard a recent book, "Rehabilitation Medicine for Elderly Patients", offers insight into the complex world of older persons in their different conditions (healthy, frail, diseased or dying) from the perspective of Physical Medicine and Rehabilitation (PM\&R), but approaching matters from a of variety of viewpoints. Written primarily for junior doctors and PM\&R residents, it is a comprehensive educational tool, simple, easy to read, clear, and written in a structured way. It may help physiatrists in reviewing the main topics in the field of rehabilitation, and also general practitioners in assisting patients with disabling conditions to solve their health problems.
\end{abstract}

World older population is rapidly growing in number and proportion and people over 60 years are expected to double in the next few decades (United Nations, Department of Economic and Social Affairs, Population Division (2015). World Population Ageing, 2015). The European continent is projected to remain for at least the next 50 years the world's area with the oldest population: by 2050 about 37 $\%$ of Europeans is expected to be 60 or over (Communication from the commission to the Council and the European parliament, 2002). Increased life expectancy which reflects, at least in part, the success of public health interventions, has many implications: the growing number of older persons suffering of chronic diseases increases demands on the public healthcare system, costs for management, and social burden. Delirium, sarcopenia, frailty, falls, dizziness, urinary incontinence are just some of the most common medical conditions that occur among the elderly, diminish their quality of life (QOL) and contribute to their physical and cognitive decline, disability and death. However, ageing itself should not be considered negatively and many interventions can be set up by the governments, communities and families in order to promote health, participation, social inclusion and security of older people and improve their QOL (Policies and priority interventions for healthy ageing - WHO/Europe, 2012). The elderly individuals, of whom $60-75 \%$ are healthy, $20-30 \%$ suffer from chronic diseases, and $2-10 \%$ are "frail" should benefit from health-related differentiated and targeted measures applied in the different contexts in which they live. It is in this regard that the book, Rehabilitation Medicine for Elderly Patients [1], offers insight into the complex world of older persons in their different conditions (healthy, frail, diseased or dying) in the specialty of Physical Medicine and Rehabilitation (PM\&R), approaching matters from a of variety of viewpoints. It represents the perspective of some international leaders in the field based on both updated literature review (including the one from biomedical and experimental approaches) and their practical clinical applications to ensure high quality standards to the book contents. Rehabilitation Medicine for Elderly Patients is organized into three parts: Part I is general and deals with many issues of elderly people such as sarcopenia, frailty, exercise, nutrition, new technologies, therapeutic modalities, etc. Part II is on rehabilitation of the most commonly encountered diseases of seniors. Some innovative topics covered in this part are cognitive therapy, spinal lesions, sexual dysfunction rehabilitation, etc. Part III is devoted to description of different organization of healthcare system for the elderly in European countries. The book is written primarily for junior doctors, who are about to embark on their studies, and PM\&R residents; therefore, it is a comprehensive educational tool, simple, easy to read, clear, and written in a structured way. However, it can also help physiatrists in reviewing the main topics in the field of rehabilitation, general practitioners and physicians of other specialties in assisting patients with disabling conditions solve their health problems. The first edition of Rehabilitation Medicine for elderly patients, like all first attempts, has limitations. Nonetheless we hope it can be appreciated as an important resource for training and education in the field of PM\&R of medical professionals and beyond.

${ }^{\star}$ Correspondence to: Ugo Carraro, Department of Biomedical Science, Interdepartmental Research Centre of Myology, University of Padova, Padova, Italy; E-mail: ugo.carraro@unipd.it

Key words: Rehabilitation, medicine, healthy elderly, frail patients Special Issue: Assisted Exercise

Ugo Carraro

Interdepartmental Center of Myology

University of Padova

Italy

Paolo Gargiulo

Inst. f. Biomed. and Neural

Engineering / Biomed Technology Centre

Reykjavik University \& Landspitali Reykjavik

Iceland

Received: May 16, 2018; Accepted: May 25, 2018; Published: June 08, 2018 


\section{Conflicts of interest}

The authors declare none conflict of interest regarding the publication of this paper.

\section{Funding statement}

None

\section{Acknowledgments}

UC thanks for support A\&C M-C Foundation for Translational Myology, Padova, Italy.

\section{References}

1. Rehabilitation Medicine for Elderly Patients, Masiero S, Carraro U, Eds. ISBN 9783-319-57405-9 ISBN 978-3-319-57406-6 (eBook) DOI 10.1007/978-3-319-57406-6.

Copyright: $\odot 2018$ Masiero S. This is an open-access article distributed under the terms of the Creative Commons Attribution License, which permits unrestricted use, distribution, and reproduction in any medium, provided the original author and source are credited. 